\title{
Marketing educacional como factor crítico de éxito en el servicio académico para alumnos universitarios
}

\author{
Educational marketing as a critical success factor in academic service for \\ university students
}

Karen Anabell Sánchez Núñez. ${ }^{1}$, Kimberlyn Mishell Vinueza Barrionuevo. ${ }^{2}$ \& Edwin Cesar Santamaría Díaz. ${ }^{3}$

DOI: https://doi.org/10.33262/visionariodigital.v5i3.1744

\begin{abstract}
.
The student area is becoming more and more competitive, it corresponds to the fact that educational institutions try to find new methods such as educational marketing to be in constant innovation and compete among universities for prestige and brand positioning in the student's mind, considering existing problems in the student service and in providing immediate solutions since the university is indifferent to retain the same, so it has generated problems with the student body. To analyze the intervention of educational marketing in the academic service to satisfy the needs of the students of the Universidad Técnica de Ambato. The research design was a qualitative approach and descriptive method. The population was 15825 students from various faculties, with a sample of 375 students of the UTA, corresponding to the academic period October 2016 - March 2017, a survey was developed through google forms, which consisted of 12 items, was approved by professionals in topics related to research. The true results obtained in the research were immersed in an analysis of external and internal variables as a priority for the academic formation of excellence and improvement with a perspective of good service as the immediate solution accompanied by empathy, trust, communication, respect,

\footnotetext{
1 Universidad Técnica de Ambato, Tungurahua, Ecuador, ksanchez4853@uta.edu.ec, https://orcid.org/0000-0001-8213-5467

2 Universidad Técnica de Ambato, Tungurahua, Ecuador, kvinueza6115@uta.edu.ec, https://orcid.org/0000-0001-6393-0246

3 Universidad Técnica de Ambato, Tungurahua, Ecuador, ec.santamaria@uta.edu.ec, https://orcid.org/0000-0003-1803-2572
} 
overcoming the expectations of students when exposed to academic problems. Among the results it was determined that the immediate student attention with $33.60 \%$, it can be confirmed that this factor is of great importance for students, the tools that should be implemented improvements are: Training and congresses with 40\%, Catalog and online repositories with $12.73 \%$, Teaching and educational activities with $8.53 \%$. Academic variables with $9.87 \%$. With a negative level of satisfaction on the part of the student body among women and men with a range of: 17 to 40 years old, for which they are considered as those opportunities for improvement, with a scale of (2 to 1). It was concluded that the integrated management model of educational marketing Migme allowed describing relevant criteria that are immersed in the opinions obtained from the respondents such as: satisfaction, exchange, facilitation, communication, intervening members, infrastructure, academic processes according to the needs of society. With the purpose of acquiring satisfactory experiences of the student body through a main factor such as loyalty through fundamental tools: learning videos or motivational videos, feedback, training, congresses, favorable infrastructure, virtual library as catalogs and repositories, laboratories with appropriate technology that serve as a benefit to obtain a favorable behavior in which students are at ease in the institutional academic period.

Keywords: Educational marketing, Academic service, Migme Model, Satisfaction, Loyalty

\section{Resumen.}

El área estudiantil es cada vez más competitiva, corresponde a que las instituciones educativas tratan de buscar nuevos métodos como el marketing educacional para estar en constante innovación y competir entre universidades por el prestigio además del posicionamiento de marca en la mente del estudiante, considerándose en la actualidad problemas existentes en el servicio de atención estudiantil y en brindar soluciones inmediatas ya que la universidad es indiferente por fidelizar al mismo, por lo que se ha generado inconvenientes con el alumnado. Analizar la intervención del marketing educacional en el servicio académico para la satisfacción de las necesidades del alumnado de la Universidad Técnica de Ambato. El diseño de investigación fue un enfoque cualitativo y método descriptivo. La población fue de 15825 estudiantes de diversas facultades, con una muestra de 375 alumnos de la UTA, que corresponde al período académico octubre 2016 - marzo 2017, se elaboró una encuesta a través de google forms, la cual constó de 12 ítems, fue aprobada por profesionales en temas relacionados a la investigación. Los resultados verídicos obtenidos en la investigación estuvieron inmersos a un análisis de variables externas e internas como prioridad para la formación académica de excelencia y mejora con una perspectiva al buen servicio como la solución de forma inmediata acompañada de empatía, confianza, comunicación, respeto, superación de expectativas del alumnado al ser expuestos a inconvenientes académicos. Entre los resultados se determinó que la Atención estudiantil inmediata con 33.60\%, se puede confirmar que este factor es de gran importancia para los alumnos, las herramientas que se deben implementar mejoras son: las Capacitaciones y congresos con un 40\%, Catálogo y repositorios en línea con $12.73 \%$, Actividades de enseñanza y educación con $8.53 \%$, 
Variables académicas con $9.87 \%$. Con un nivel de satisfacción negativo por parte del alumnado entre mujeres y hombres con rango de: 17 a 40 años, para para lo cual se consideran como aquellas oportunidades de mejora, con una escala de (2 a 1). Se concluyó que el modelo integrado de gestión de mercadeo educativo Migme permitió describir criterios relevantes que están inmersos en las opiniones obtenidas por parte de los encuestados como: satisfacción, intercambio, facilitación, comunicación, miembros intervinientes, infraestructura, procesos académicos según las necesidades de la sociedad. Con la finalidad de adquisición de experiencias satisfactorias del alumnado a través de un factor principal como la fidelización mediante herramientas fundamentales: videos de aprendizaje o videos motivacionales, feedback, capacitaciones, congresos, infraestructura favorable, biblioteca virtual como catálogos y repositorios, laboratorios con tecnología adecuada que sirvan de beneficio para la obtención de un comportamiento favorable en el cual los estudiantes se encuentren a gusto en el período académico institucional.

Palabras claves: Marketing Educativo, Servicio Académico, Modelo Migme, Satisfacción, Fidelización.

\section{Introducción.}

La Universidad Técnica de Ambato es una institución de educación superior, que debería desarrollar técnicas nuevas que generen estrategias para ser una de las mejores instituciones, sin embargo, aún no logra establecerse en la mente del consumidor a nivel nacional.

El marketing educativo permite mejorar aquellos aspectos críticos de éxito para conseguir la satisfacción en los requerimientos estudiantiles además de lograr un posicionamiento a nivel nacional por su servicio y también por las estrategias practicantes

Se sustenta esta investigación con varias teorías para establecer ideas claras que aporten al desarrollo de estrategias por medio del marketing educativo, es así como la investigación permite realizar el análisis de datos para generar propuestas enfocadas en la calidad, satisfacción y fidelización como mejora de expectativas.

\section{Educación Superior}

El autor (Tzu, 2016) enfatiza que las universidades están inmersas a un nivel competitivo el cual es destacado con respecto al incremento de oferta académica, por ello es fundamental que la educación superior analice y seleccione estrategias que sirvan como mejora en relación a sus competidores además de garantizar un posicionamiento duradero y generación de valor de marca.

La educación superior es considerada como una herramienta importante, por su alto nivel de conocimiento, servicio académico, es así como la institución debe ser de calidad para generar una ventaja competitiva que interese para el desarrollo personal y aprendizaje significativo en los estudiantes universitarios (Andrade, 2017). 
Las instituciones de educación superior implementan nuevos métodos de enseñanza y atención de requerimientos que esté expuesto el estudiante. El posicionamiento en la mente de los alumnos actuales y potenciales, permite lograr prestigio en el ámbito académico (Parmerlee, 2017).

El autor (Pastor, 2017) alude que la oferta académica se forma de acuerdo a las necesidades sociales, efectivamente el aprendizaje es considerado como un servicio, es decir la formación debe implementar herramientas de mejora constante para lograr la fidelización.

(Rué, 2018) deduce que las instituciones de educación superior optan por competir y mejorar sus modalidades de enseñanza e implementar nuevos métodos que permitan brindar un servicio educativo de calidad, dado que el estudiante es un cliente al cual se debe fidelizar a través de la facilitación de información para su conocimiento.

El autor (Sanz M. L., 2017) menciona que la facilidad de generar una oferta académica es un intermediario entre el intercambio y el costo el cual actúa como progreso de las necesidades que posee el estudiante. En las instituciones de formación superior prevalece el valor de marca de manera cualitativa al mencionar los aspectos positivos, es decir la inscripción de matrícula, derecho de grado, becas, transporte domiciliario con gratuidad que incentiva al alumnado a no tener limitaciones de aprendizaje y formación.

Para reestructurar la oferta académica se plantea como sugerencia que a través de reuniones en las instituciones mejoren su aspecto de utilidad, es decir plantear estrategias que permitan reformar el sistema educativo para el cambio de ideales antiguos por otros modernos al mismo tiempo establecer programas para el proceso (Barcia \& Cevallos, 2019).

(Ruiz, 2017) Deduce que la educación superior es considerada como una herramienta de capacitación para que el personal brinde un servicio estudiantil de eficacia, en efecto interviene tanto directivos como personal administrativo, además existe otro tipo de personal que interactúa de forma directa como son los docentes, todos aquellos deben poseer características y conocimiento que aporte de ayuda al estudiante.

El autor (Escobar, 2016) detalla que la investigación permite indagar, analizar para la adquisición de nuevos conocimientos y desarrollo de capacidad de búsqueda e innovación, es importante que las instituciones educativas estén aptas en diferentes aspectos como la implementación de estrategias en la solución de posibles inconvenientes.

El autor (Gonzalez, 2016) deduce que las instituciones de educación superior poseen un enfoque académico ideal para desarrollo de calidad en el servicio estudiantil. La oferta académica prepara a los jóvenes universitarios al desenvolvimiento en el área profesional de hecho, las instituciones son las que mayor demanda tienen y al mismo tiempo permiten conseguir la acreditación de carreras universitarias, mientras que si no estructura de forma correcta su servicio pueden perder la categoría. 


\section{Satisfacción del Alumnado}

El autor (Magro, 2015) define que el alumnado manifiesta su satisfacción a través del bienestar estudiantil como son: los servicios de aprendizaje, interacción, retroalimentación, buen ambiente laboral que demuestre compañerismo y equidad con ayuda de un equipamiento necesario para el desenvolvimiento del estudiante adecuado que permita el desenvolvimiento de aptitudes y destrezas.

Según (Kotler, 2015) menciona que los usuarios universitarios son los más idóneos para evaluar el servicio que la universidad emite, las valoraciones son de forma parcial que ayudan para el análisis de indicadores en el mejoramiento de la educación superior de calidad acoplada al progreso y gestión de programas académicos.

Según (Bassat, 2016) deduce que el rendimiento académico se asocia con la satisfacción del alumnado, por ende intervienen las emociones de un individuo frente al cumplimiento de las expectativas enfocadas al éxito, además de conseguir la acreditación que contribuye como un elemento importante para el prestigio de marca.

El autor (Kawasaki, 2015) manifiesta que es importante descifrar y entender como los clientes universitarios perciben su organización, además de proponer metas para lograr el mejoramiento de servicios académicos acompañado de un seguimiento periódico con la finalidad de mantener los beneficios con avances constantes para conseguir la fidelización del alumnado.

El autor (Larios, 2015) alude que la mercadotecnia es un instrumento que satisface necesidades de los estudiantes, ya que es considerado como un intercambio, además se presenta de forma presencial o virtual, cabe destacar que el servicio educativo interviene en diversos aspectos como: intangibilidad, actividad inseparable, heterogéneo, perecedero.

El autor (Belío, 2016) expresa que debido a la demanda de estudiantes, las instituciones optaron por mejorar sus servicios académicos, se estima que el estudiante es como el cliente en una institución, es fundamental brindar las mejores estrategias y técnicas para que el cliente pueda obtener el mejor servicio estudiantil, al mismo tiempo será recompensado por el prestigio que obtendrá la institución.

La investigación es un factor que aporta al mejoramiento de calidad de vida como énfasis en la superación de los individuos, además de asumir retos de un progreso satisfactorio a docentes y estudiantes para la contribución del progreso de la sociedad y sostenibilidad (Aldamiz, 2016).

Es necesario atender las sugerencias y requerimientos para que el estudiante pueda obtener una atención de calidad y satisfacer su interés por lo solicitado, además una buena atención genera comodidad, conformidad. Es significativo generar preferencia del estudiante por la institución académica, el agrado del servicio otorgado ayuda a los estudiantes en general como proceso de lealtad debido a la solución asignada (Tobón, 2016). 
Según (Paris, 2015) detalla que la universidad necesita fomentar una relación agradable en la atención de los servicios académicos con los estudiantes para generar motivación y satisfacción al recibir un trato agradable además de la solución de necesidades.

Cada institución posee un programa académico el cual está enlazado con calidad que conforman docentes, estudiantes, administrativos, por lo tanto, deben estar aptos para desempeñar sus funciones de acuerdo a sus competitividades. La experiencia de un estudiante involucra sentimientos, emociones, recuerdos como procesos de su aprendizaje, es decir que la experiencia de un cliente no se centra solo en cambios de cultura para fomentar ingresos, sin embargo desea llegar a los individuos (Llorente, 2019).

La cultura de hoy en día varia, las instituciones son más preparadas para brindar su servicio académico, el cliente se fideliza de acuerdo a la cantidad de emociones transmitidas al recibir un buen servicio, por ende las instituciones reestructuraron su enfoque en la mejora de servicios (Ferré, 2017).

Algunos factores que ayudan como motivación a la excelencia académica y a la satisfacción son: reconocimiento a docentes, estudiantes destacados, convenios con países internacionales para desempeño de conocimiento en prácticas pre profesionales, servicios de bienestar universitario como; atención en el área médica, psicológica, cultural. A causa de la gran cantidad de estudiantes en los centros educativos, se puede inferir que no existía preocupación por brindar un servicio de calidad en la educación, sin duda alguna este nuevo siglo es un generador total de cambios ideológicos y en construir la excelencia (Almanza \& Ustate, 2018).

\section{El Marketing Educativo}

El autor (Sarrias, 2018) indica que el marketing educativo es una herramienta que permite investigar e indagar necesidades para elaborar un proceso de mejora en el desarrollo de nuevos conocimientos, por ello debe ser realizado en un lugar y tiempo acordado. Este aspecto del marketing se da cuando las organizaciones educativas se preocupan por mejorar su calidad en la educación, esto se efectúa cuando existe más demanda en las universidades y preferencia por el reconocimiento, como resultado lleva a tomar otro tipo de estrategia para convertirla en una mejor opción.

El autor (Sanz, Crissien, García, \& Patiño, 2016) deducen que el marketing en la educación se involucra en la investigación de necesidades que satisfagan a los estudiantes, es decir se desenvuelve mediante un análisis de la institución, como puede ser un modelo en la calidad que permita desarrollar una ideología tanto de la expectativa como de la percepción del servicio que brinda la institución.

(Cárdenas, 2015) detalla que la función del mercadeo educativo ayuda a las instituciones a cumplir con los requerimientos plantados, además ayuda a obtener un análisis para realizar un cambio de mejora, el marketing educacional se enfoca en mejorar la calidad 
en los servicios de acuerdo a la toma de decisiones, la acción seleccionada se involucrada directamente o indirectamente a la comunidad estudiantil.

El autor (Jiménez, 2015) menciona que el plan de mercadeo educativo recopila diferentes puntos como: la situación actual, determinación de objetivos, elaboración de estrategias, programas y presupuestos, evaluación y control. Este plan ayuda a fomentar estrategias a base de la necesidad actual de la institución correspondiente.

(Meneses, Muñoz, Cantos, \& Freire, 2018) aluden que lo impactante de la mercadotecnia en la educación es la posible propuesta de una institución, además hoy en día existe instituciones que consideran al estudiante como un problema, de modo que se evade la opción de cliente, en oferta educativa por falta de intereses en la comunidad estudiantil.

El autor (Dvoskin, 2016) considera que para desarrollar el marketing educativo en las instituciones se debe reestructurar un nuevo enfoque que permitirá establecer ideas claras de cómo mejorar los procesos académicos y brindar una mejora en servicios, algunas instituciones optan por reuniones para innovar de ideas e intercambiar opiniones a fin de lograr formar una sola ideología.

El autor (Mamani, 2016) expresa que el marketing educativo pretende tener una buena relación entre la institución con el estudiante con el propósito de fidelizar y recuperar a estudiantes perdidos, además busca escuchar sus necesidades para presentar una solución de forma inmediata y estar en constancia lo que proporcionará brindar un servicio de calidad al alcanzar la mayor experiencia en los estudiantes. Por consiguiente, el autor destaca dos áreas básicas que es gestión estudiantil y gestión en su lealtad.

Según (Hacker, 2017) manifiesta que la implementación del marketing educacional actúa como factor importante de motivación hacia el estudiante en el ámbito académico, además ayuda a estructurar una estrategia que permita dar a conocer las ventajas que posea la institución.

El autor (Bid, 2016) analiza que los servicios que se brinda al estudiante son de forma intangible, aquellos aspectos que no se pueden observar y son complejos al momento de controlar como:

- Fidelización

- Motivación

- Percepción

- Necesidades

- Actitudes

(Soriano, 2017) señala que el marketing educativo es de suma importancia, ya que permite que la institución se destaque y desenvuelva de mejor forma, además la experiencia del estudiante permitirá la fidelización, a fin de beneficiar a la institución en el prestigio que el mismo estudiante dará a conocer a otros posibles clientes. 
El marketing educacional es una mezcla del empresarial y el social, es un cambio de estructura, las personas encargadas pueden ser directores, como personal que intervenga en el servicio académico, es necesario que el mismo posea conocimiento y realice una excelente coordinación de procesos de lo contrario no existe marketing (Núñez, 2017).

El marketing educativo se basa en requerimientos necesarios para la comunidad universitaria, a través de una gestión exitosa, por lo cual se basa de acuerdo a un esquema, en caso de haber incertidumbres, por ende, permitirá a la institución actuar de forma inmediata, y como resultado el estudiante transmitirá mayor seguridad. (Tejada \& Reyes, 2015).

Para mejorar el nivel educativo se puede proponer un modelo de gestión a fin de comprender la necesidad de cambiar ámbitos actuales que mejore el servicio educativo mediante el mercadeo, de modo que abarque las $4 p$ del enfoque hacia el cliente; en este caso los estudiantes puede ser una herramienta muy útil para reestructurar el modelo, además se puede utilizar un modelo integrador que permita identificar dimensiones de mejora, a fin de lograr una educación de calidad (Pérez, 2018).

El autor (Grande, 2017) deduce que cada institución tiene diversas formas de perspectiva como son: las ofertas académicas, valores reconocimiento de imagen, diferenciación, docentes capacitados, investigadores, entre más. Las instituciones optan por realizar de la mejor forma su programa de mercadeo para no ser desacreditadas o tener una mala categoría de educación, además el plan de mercadeo ayuda a que la institución transmita el tipo de enseñanza que tiene su enfoque en mejorar la calidad de la educación y la garantía de la oferta académica.

Las instituciones anteriormente eran constantes no poseían problema y la mayoría tenían el mismo tipo de enseñanza, en la actualidad se busca mejorar la calidad, dar nuevas soluciones a los nuevos problemas sociales, es necesario enfocarse en formar un programa de mercadeo educativo adecuada para un mejorar el desarrollo de la oferta académica (Knight, 2017).

Las instituciones educativas brindan un servicio por lo cual, no se puede tocar, pero por medio del mismo se adquiere conocimiento, el marketing educativo actúa con estas instituciones para que el estudiante genere satisfacción por la experiencia otorgada en su servicio académico, también permite que el estudiante genere satisfacción al trasmitir distintos tipos de emociones que pueden ser de forma positiva o negativa, la institución debe tratar que la mayor parte sean respuestas positivas y en caso de existir negativas, debe buscar una solución inmediata, esto generará que el estudiante se sienta cómodo y conforme (Barreto, 2016).

Para (Suysuy \& Taira, 2018) concuerda que el marketing hoy en día es competitivo y da paso a que las instituciones mejoren sus métodos de enseñanzas e innoven constantemente para que la servicio otorgado al estudiante sea de forma eficiente, las instituciones deben estructurarse y tratar de estar en la mente del estudiante para obtener un espacio en el mercado. 


\section{VARIABLES DEL MARKETING EDUCACIONAL}

Según (Rocafuerte, 2016)indica que las variables en el aspecto de marketing educacional son significativos para crear valor de marca en las instituciones de educación superior como:

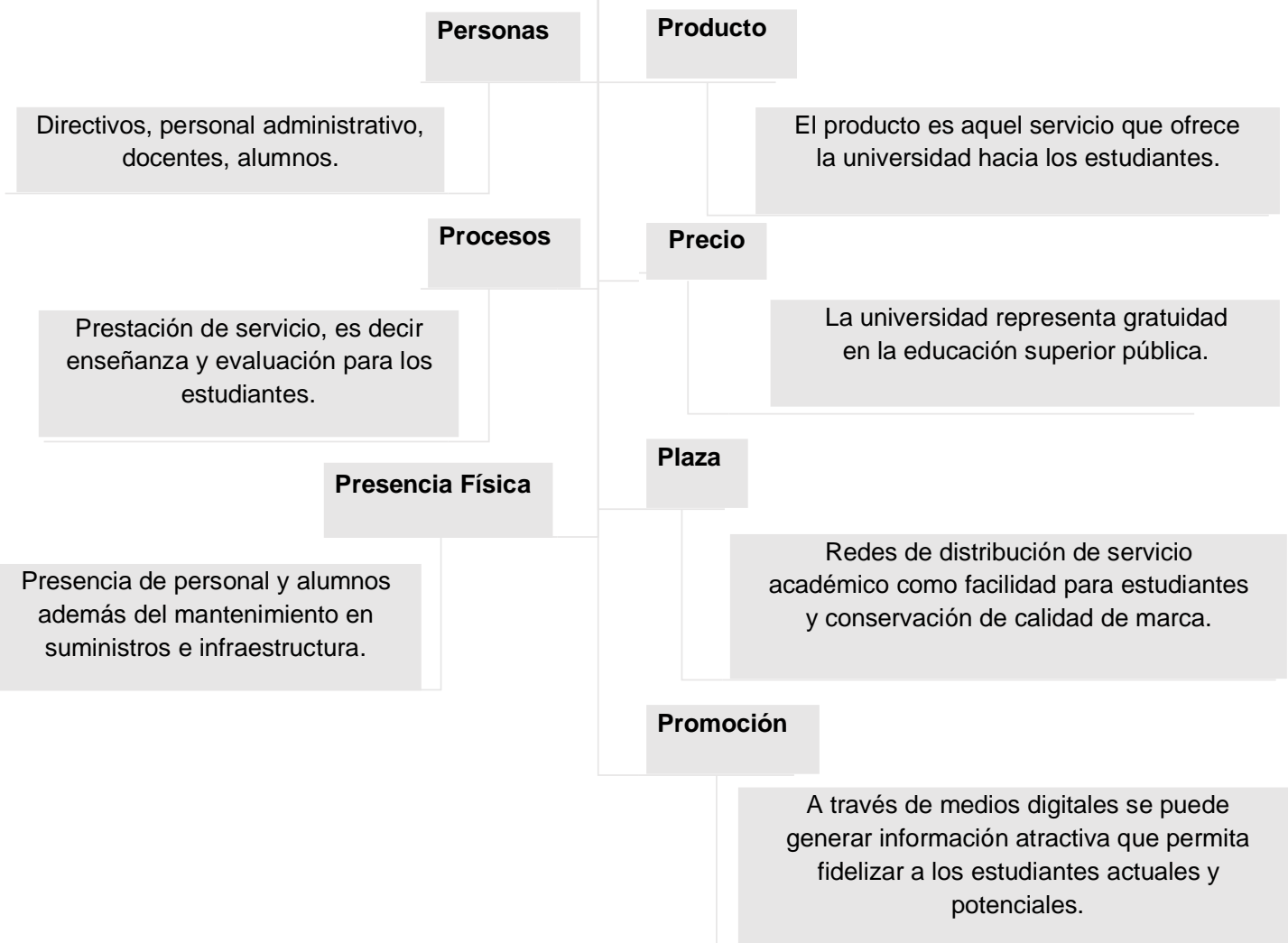

Tabla 1. Variables de Marketing Educacional

Fuente: Elaboración propia.

\section{MIGME- Modelo de Mercadeo Educativo}

El autor (Ospina \& Sanabria, 2010) detalla:

Este modelo permite realizar un análisis de variables tanto internas como externas de una institución de acuerdo a su servicio educativo.

Este modelo se complementa con los siguientes criterios:

- La satisfacción del cliente: para llegar a obtener este grado de percepción, el servicio en la parte académica es referente a su calidad, además interviene varios factores como docentes, convenios, infraestructura, titulo, bienestar estudiantil, entre otros. 
- La satisfacción del estudiante, es un proceso donde se verifica la necesidad y los requerimientos necesarios para proceder a la oferta académica, es decir que primero se estudia requerimientos para proceder realizar un análisis interno y cubrir aquellas necesidades.

- El intercambio: Es el valor que intercambia el estudiante a la institución para obtener una educación de calidad, no solo se suman los costos internos referentes a la institución, también interviene los externos como es la movilidad, entre otros. En el caso de las instituciones públicas el estado interviene en la responsabilidad de asumir este costo.

- Facilidad de acceso: En este proceso se define la facilidad que una institución brinda a los estudiantes para facilitar el acceso a su educación, a fin de intervenir en varios factores como: convenios e instalaciones que el estudiante pueda acceder para obtener la información requerida, además también interviene las modalidades que oferta cada institución como son presenciales y semipresencial.

- Comunicación: Se enfoca en las estrategias como la generación de programas académicos que beneficie el bienestar estudiantil y permita mejorar la imagen de la institución a través de canales de publicidad como internet, televisión, radio, revistas, eventos, ferias.

- Personal de intervención: Se enfoca los miembros intervinientes para brindar este servicio académico, de allí se desarrollarán características para identificar calidad, en este proceso pueden intervenir docentes, directivos, estudiantes, funcionarios, entre más, esto se considera por el rol que desempeñe con la prestación del servicio

- Proceso educativo: Este criterio se basa en actividades que desempeñe la institución para poder estructurar, analizar y evaluar su servicio académico, determinando necesidades determinada con el uso del servicio, para garantizar la calidad también interviene las ISO, programas académicos, entre más

- Infraestructura de la institución: Este criterio refleja todas las eras que proporciona la institución para un mejor desempeño, existen tangibles como es el aula de estudios, estructura propia para aspectos académicos y la intangible que se basa en el clima de trabajo, cultura, aspectos digitales, programas, entre más.

\section{Factores Críticos de Éxito en el Servicio Académico}

El autor (Belío, 2016) alude que los factores críticos de éxitos sirven de apoyo para que las instituciones logren alcanzar el nivel de satisfacción además de generar resultados exitosos conjunto con variables como: 


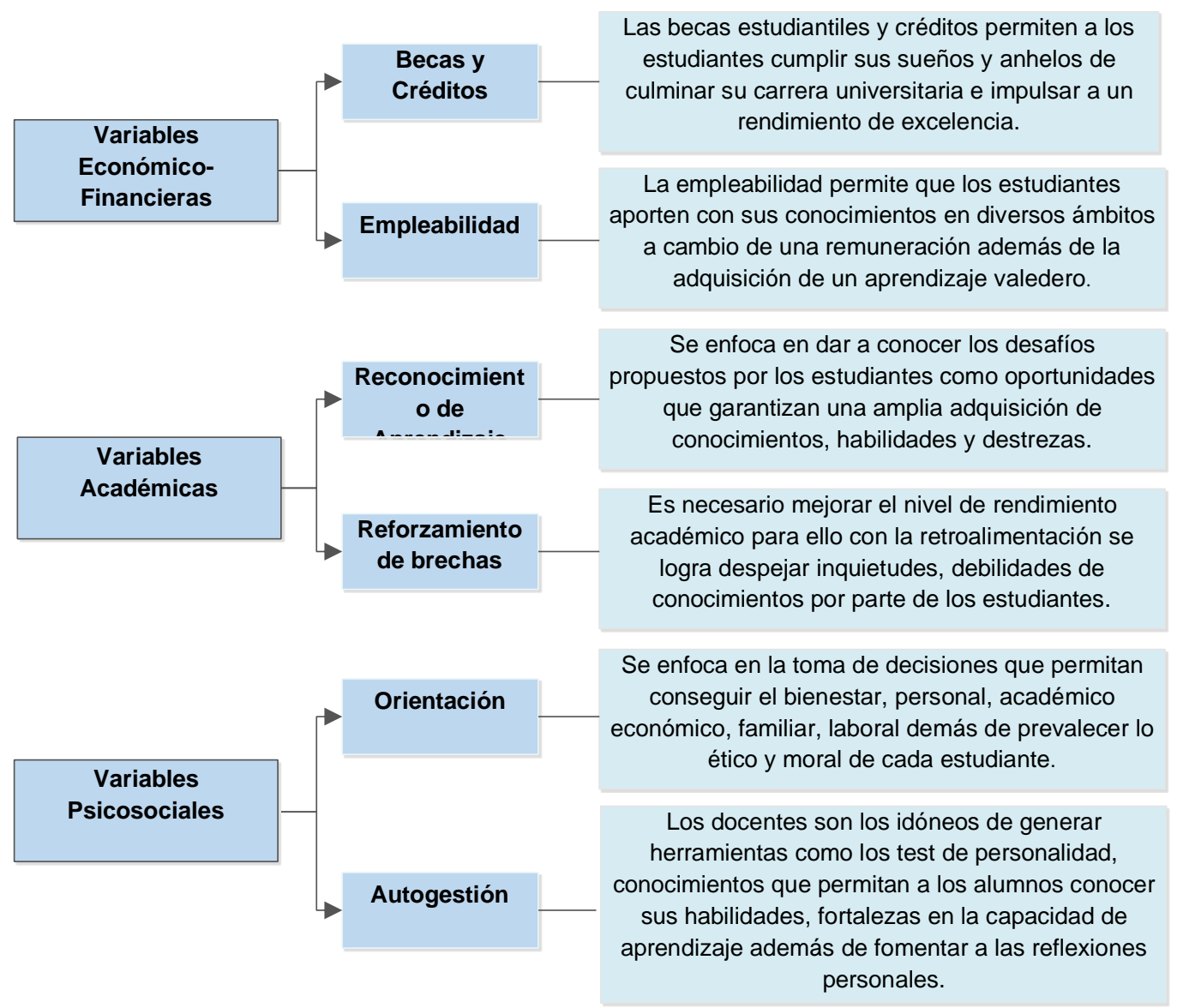

Tabla 2. Factores Críticos Éxito

Fuente: Elaboración propia.

\section{Metodologia.}

La siguiente investigación se conforma por 15825 estudiantes de la Universidad Técnica de Ambato con período académico octubre 2016 - marzo 2017 dividido por diferentes facultades correspondientes a: Ingeniería Civil y Mecánica, Diseño Arquitectita y Artes, Ciencia e Ingeniería en Alimentos, Ciencias Humanas y de la Educación, Ciencias Agropecuarias, Ciencias Administrativas, Contabilidad y Auditoría, Jurisprudencia y Ciencias Sociales, Ingeniería en Sistemas- Electrónica e Industrial y Ciencias de la Salud; establecida por áreas de: nivelación, pregrado, posgrado; que corresponde a la población objeto con un $95 \%$ nivel de confianza y un 5\% de error. El total de la muestra es de 375 estudiantes de la Universidad Técnica de Ambato, la edad seleccionada corresponde a un rango de 17 a 40 años, el género se distribuye al 50\% masculino y 50\% femenino.

El método descriptivo aplicado se basa en un enfoque cualitativo, ya que permite resaltar respuestas concretas de la satisfacción del alumnado, además de describir, rescatar las características relevantes de la investigación.

El instrumento utilizado para la recopilación de datos es la encuesta, la misma que se estableció con varios tipos de preguntas, con el fin de obtener respuestas verídicas que sirvieron como aporte beneficioso para la investigación. 
El análisis cualitativo se realizó de acuerdo a los resultados obtenidos en cada una de las preguntas del instrumento aplicado (encuesta), además se elaboró en el programa Google forms, ya que permite obtener respuestas rápidas y al mismo tiempo recopila datos automáticamente en un formulario, aporta una opción de descarga, también se aplicó por la mayor facilidad de llegar a los estudiantes de forma online, evita demoras y se obtiene mejores resultados en un tiempo real.

Para el desarrollo de la investigación se inició con la formulación del problema, recopilación de información a través de fuentes bibliográficas, ejecución de hipótesis, formulación de preguntas a la población estudiantil, análisis de resultados, además de emitir una discusión pertinente y conclusiones relevantes.

La investigación realizada cumple con todos los estándares éticos desde su planificación, desarrollo y evaluación emitida a la población estudiantil con libertad de opinión, los datos personales son confidenciales no expuestos a inconvenientes académicos.

\section{Resultados.}

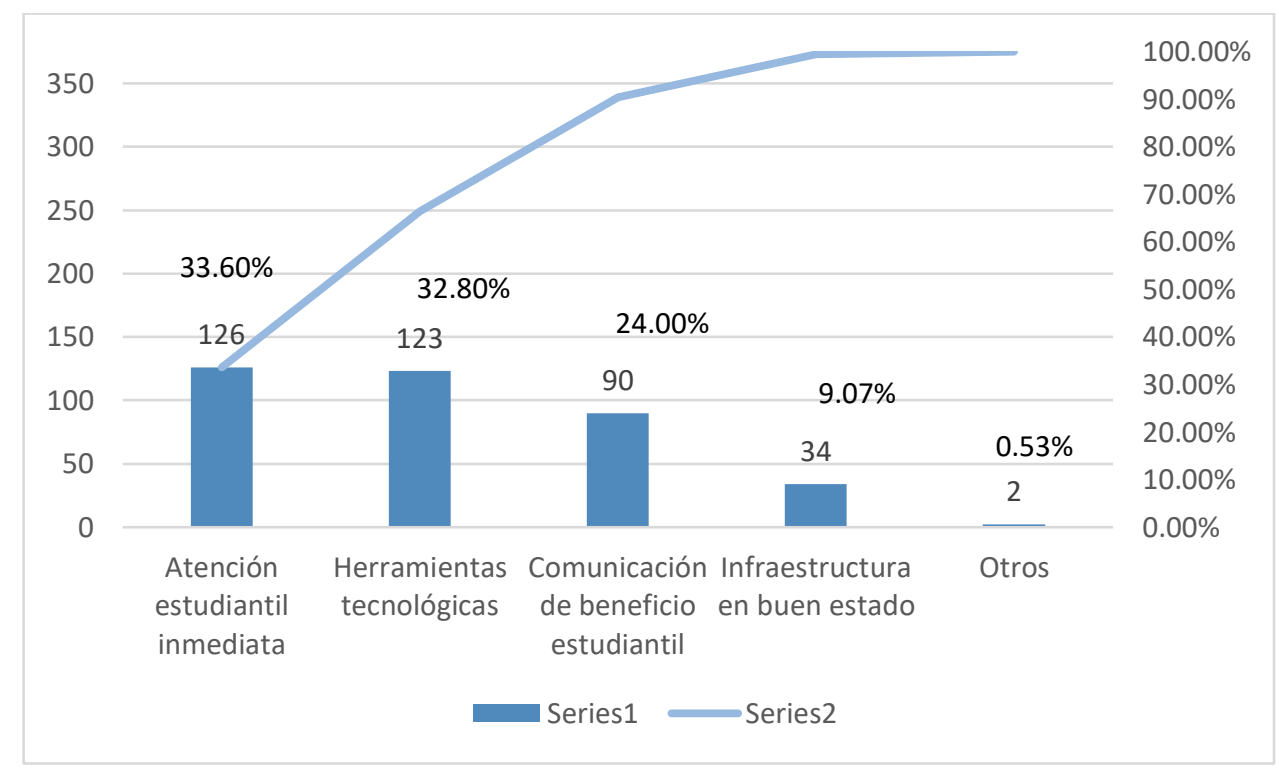

Figura 1. Factores del Servicio Académico Fuente: Elaboración propia.

De acuerdo a los datos obtenidos, el factor académico con mayor relevancia en los estudiantes es la Atención estudiantil inmediata, el cual obtuvo un resultado de 126 votos, con un porcentaje de $33.60 \%$, por lo tanto se puede confirmar que este factor es de gran importancia para los alumnos y es necesario determinar el grado de satisfacción del mismo, las herramientas tecnológicas ocupan el segundo lugar con un total de 123 votos, equivalente al $32.80 \%$, el factor intermedio es la Comunicación de beneficio estudiantil con 90 votos, correspondiente al $24 \%$ y los factores con menor importancia es la Infraestructura en buen estado con 34 votos, que corresponde al 9.07\% y Otros con 2 votos, con un resultado de $0.53 \%$. 


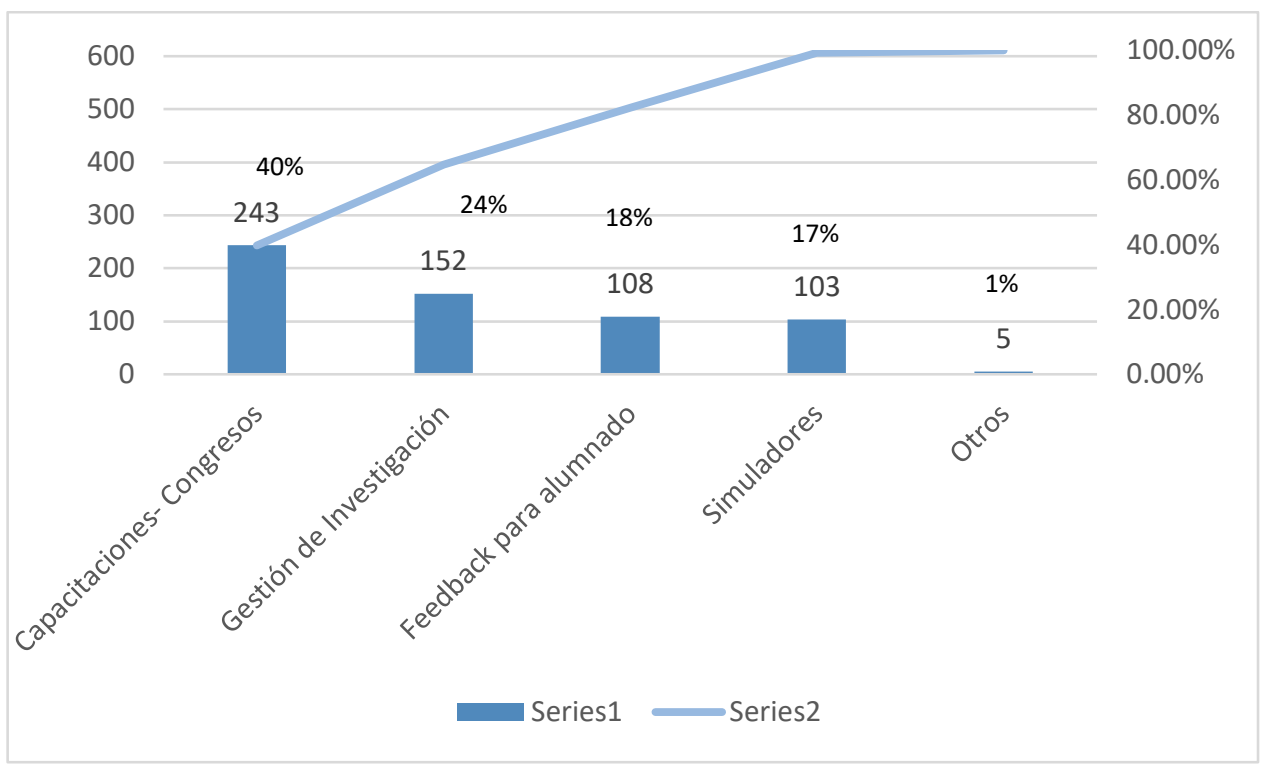

Figura 2. Herramientas satisfactorias de las necesidades del alumnado Fuente: Elaboración propia.

Según datos arrojados la perspectiva de los estudiantes en cuanto a las herramientas que se deben implementar mejoras son: las Capacitaciones y congresos, el cual obtuvo un resultado de 243 votos, con un $40 \%$ al ser el mayor factor en liderar en las herramientas satisfactorias de las necesidades del alumnado, el cual se confirma el elevado grado de insatisfacción, para ello a través de la implementación de estrategias de mercadeo se requiere mejorar la percepción de los universitarios. Por consiguiente, se encuentra la parte de Gestión de investigación con un total de 152 votos y un $24 \%$ mientras que con un valor intermedio se encuentra Feedback para alumnado de 108 votos y un $18 \%$ continuado de Simuladores con 103 votos y 17\% con respecto a Otras herramientas se obtuvo tan solo 5 votos que equivale al $1 \%$.

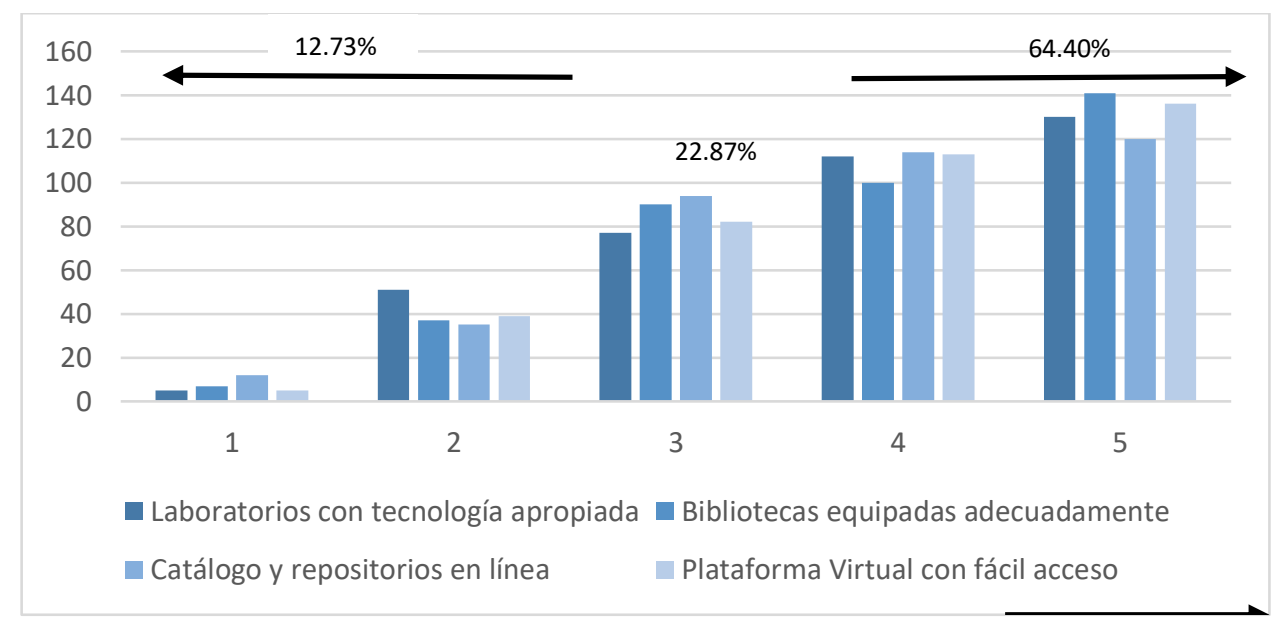

Figura 3. Herramientas que ayudan el proceso de formación educativa Fuente: Elaboración propia. 
Según datos obtenidos se puede analizar que los estudiantes ratifican que dentro de las herramientas que ayudan a la formación educativa, a partir de izquierda a derecha con una escala de (4 a 5) corresponde al $64.40 \%$ con un nivel de satisfacción positivo para Bibliotecas equipadas adecuadamente esto quiere decir que se debe mantener, mientras que a partir de derecha a izquierda con una escala de (2 a 1) corresponde al $12.73 \%$ con un nivel de satisfacción negativo para catálogo y repositorios en línea para lo cual se consideran como aquellas oportunidades de mejora. Por otro lado, los estudiantes universitarios consideran la escala (3) que corresponde al $22.87 \%$ con un valor intermedio de satisfacción para catálogos y repositorios en línea, con tendencia a un valor negativo similar a la escala anterior.

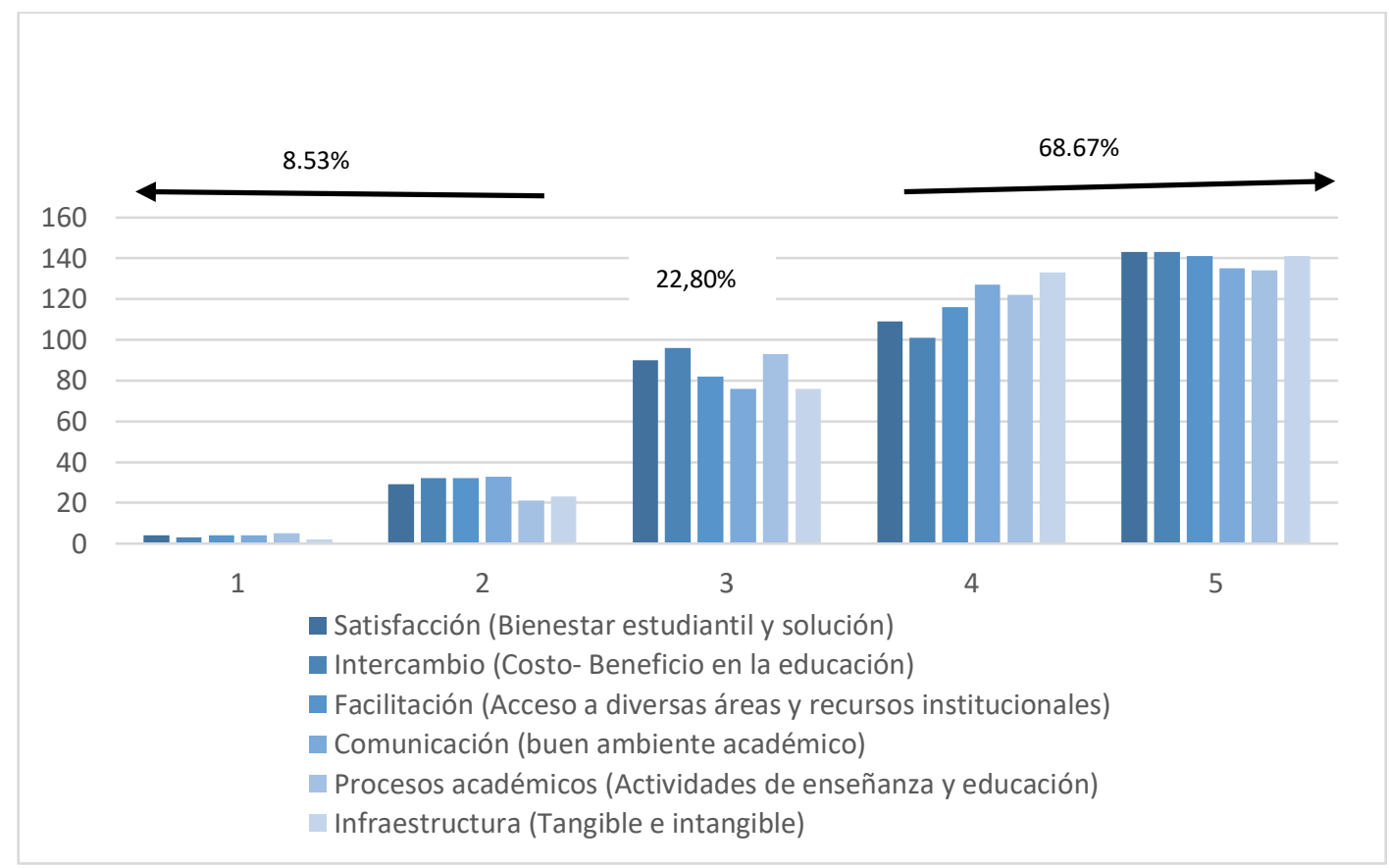

Figura 4. Plan de mercadeo educativo MIGME Fuente: Elaboración propia.

Según los puntos más relativos que los estudiantes consideran importantes al analizar y mejorar en relación al modelo plan de mercadeo educativo MIGME, de referencia se observa que a partir de izquierda a derecha con una escala de (4 a 5) corresponde al $68.67 \%$ con un nivel de satisfacción positivo para el Bienestar estudiantil y solución al igual que Costo-Beneficio en la educación, esto quiere decir que se debe mantener, mientras que a partir de derecha a izquierda con una escala de (2 a 1) corresponde al $8.53 \%$ con un nivel de satisfacción negativo para las Actividades de enseñanza y educación por lo tanto se consideran como aquellas oportunidades de mejora porque también son necesarios para la implementación de herramientas de mejora y así lograr una ventaja competitiva para la Universidad Técnica de Ambato. Además, el alumnado manifiesta que la escala (3) corresponde al $22.80 \%$ con un valor intermedio de 
satisfacción para el intercambio de Costo-Beneficio en la educación, con tendencia a un valor positivo como la primera escala.

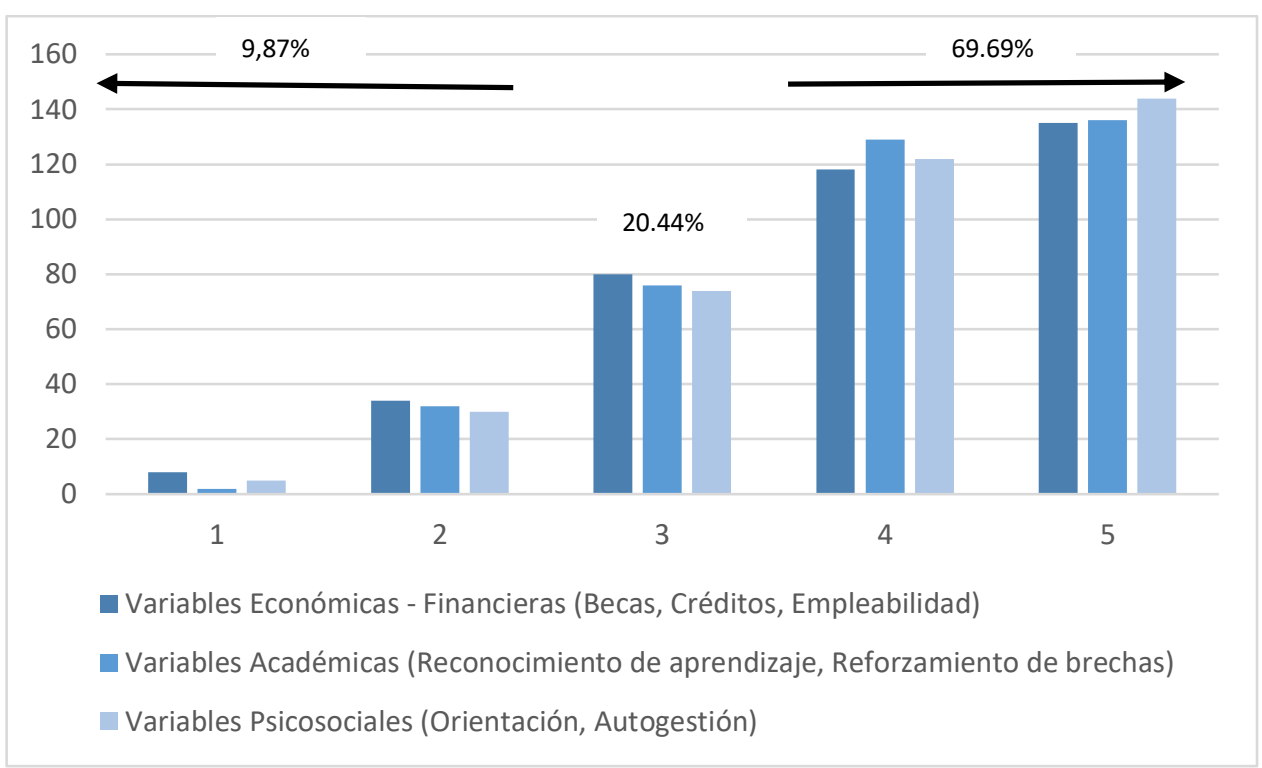

Figura 5. Factores críticos de éxito en la Educación Superior Fuente: Elaboración propia.

Según los datos obtenidos, los estudiantes establecieron su grado de importancia, respecto a los factores críticos éxito en la Educación Superior, de izquierda a derecha se determina el aspecto positivo, correspondiente a la escala (4-5) con un resultado 69.69\%, el cual se puede concluir que la mayoría de los estudiantes consideran que los factores mencionados son de gran relevancia en la Educación Superior, a partir de derecha a izquierda se establece el aspecto negativo, correspondiente a la escala (2-1) con un resultado $9.87 \%$, los estudiantes concuerdan que los factores críticos mencionados no aportan de forma esencial en la Educación Superior y el $20.44 \%$ interviene con un grado de importancia neutro. 
Tabla 3: Modelo de Gestión Educativa

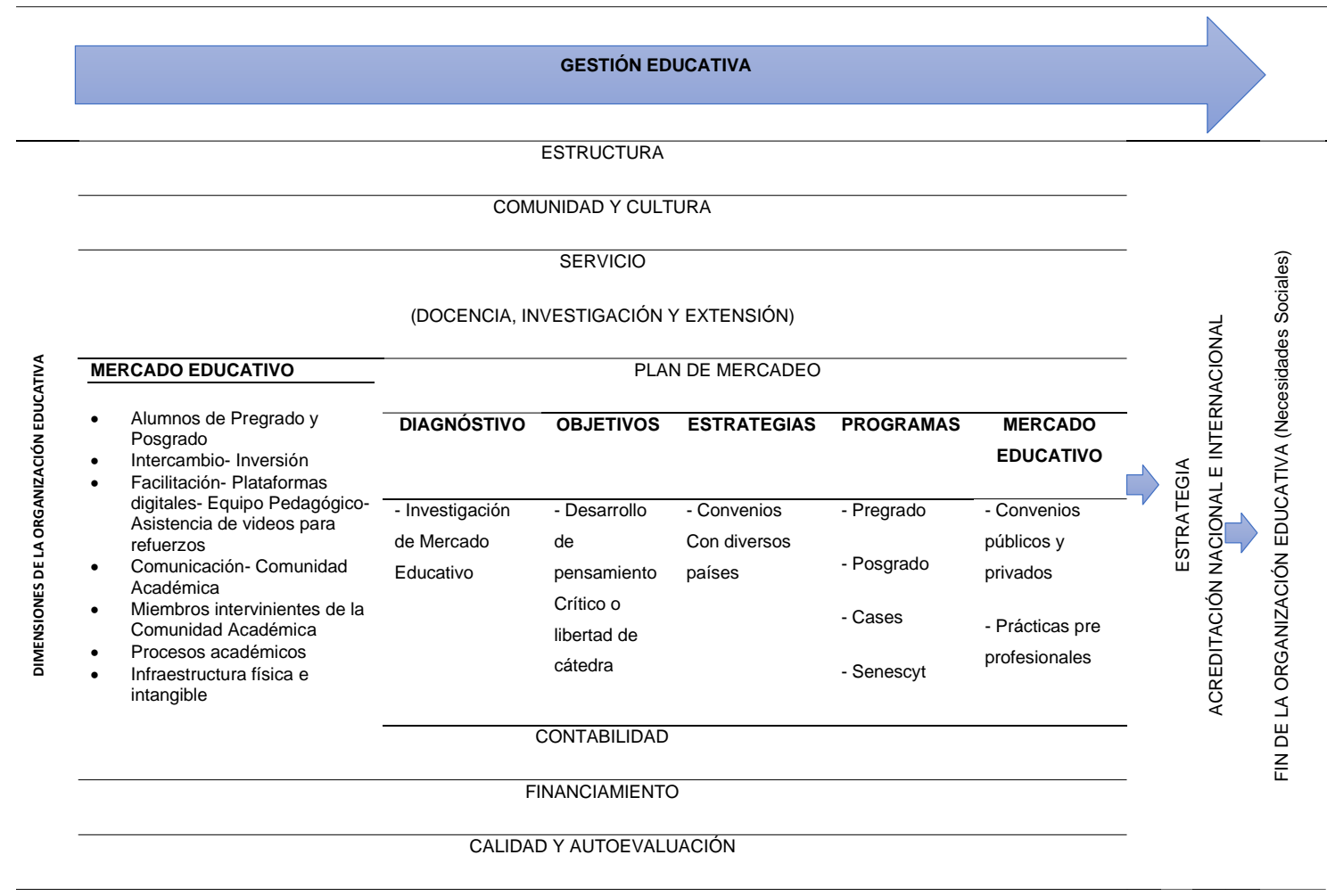

Fuente: Elaboración propia.

El siguiente modelo de gestión educativa, es una estructura que permitirá realizar cambios de mejora en la educación, el cual se basa en 7 dimensiones educativas que son: Estructura, Comunidad y Cultura, Servicio, Plan de mercadeo, Contabilidad, Financiamiento, Calidad y Autoevaluación. En la parte de estructura se establecerá todo lo referente a la parte interna como externa de la Universidad Técnica de Ambato, en el cual consta también el equipamiento de laboratorios y herramientas necesarias para la facilitación de estudio y de esta manera el alumnado se encuentre cómodo y satisfecho. Comunidad y Cultura, se basa en la buena imagen y en los valores que la institución debe llevar a cabo y dar cumplimiento, la misma debe ser realizada por todos los miembros que conforman la comunidad universitaria. Servicio, esta parte se basa al equipo que debe determinar una labor, ya sea en la parte de docencia, en investigación u otras áreas que sean necesarias para la formación académica del estudiante. Plan de Mercadeo, está establecido por varios pasos, como primer punto se realizará un diagnóstico, se desarrollarán objetivos, estrategias, programas y por último se establece el mercado educativo, luego de establecer estos pasos se procede a analizar herramientas de mejora para la generación de estrategias y de esta manera conseguir la acreditación, la misma que puede ser de forma nacional o internacional. Por consiguiente, la quinta dimensión es la parte contable la cual se encarga de la estructuración de los estados financieros con la finalidad de emitir reportes entre ingresos y egresos que la Universidad genera en el transcurso de sus periodos académicos además de emitir excelentes tomas de decisiones que favorezcan la rentabilidad de la institución. Otro factor es el financiamiento de los 
estudios universitarios, el cual se considera importante porque permite destacar el excelente rendimiento académico a través de becas y créditos. La última dimensión es la calidad y autoevaluación, esta enfrenta diversos desafíos por la Educación Superior porque permite saber el nivel de aprendizaje y de esa forma mejorar a través de la retroalimentación.

\section{Discusión}

Los estudiantes determinaron que los factores con mayor grado de importancia en el Servicio Académico (Figura 1), están entre la Atención estudiantil inmediata con un resultado del $33.60 \%$ y las herramientas tecnológicas con el $32.80 \%$, esto se debe a que los alumnos de la Universidad Técnica de Ambato tratan de organizar su tiempo y no consideran correcto desperdiciarlo en largas esperas, por otro lado, las herramientas tecnológicas ayudan a su desarrollo académico, como un factor de suma importancia en su educación. En la parte intermedia se encuentra la Comunicación de beneficio estudiantil con un $24.00 \%$, esto se debe a que este factor no beneficia a todos de estudiantes y con menor grado de importancia esta la Infraestructura en buen estado con un $9.07 \%$ y Otros factores con $0.53 \%$, ya que no afectan directamente al desempeño estudiantil.

De acuerdo a los resultados como se observa en la (Figura 2) se debe a un inmediato fortalecimiento de Capacitaciones y congresos que corresponde a un nivel de percepción mínimo por parte del alumnado con un $40 \%$, estos pueden ser de mayor relevancia de acuerdo al tipo de carrera o a su vez que aporte hacia su nivel de estudio y como la contribución de requisitos laborales que avalen su formación. Para ello se puede basar en el instrumento del modelo educacional MIGME, el cual permitirá establecer una estructura de lo que se tiene y lo que se pretende alcanzar, con ayuda de estrategias para la mejora de las herramientas satisfactorias de las necesidades del alumnado y conseguir el nivel máximo en cuanto a bienestar. Además, el nivel grado intermedio de satisfacción es Feedback para alumnado con un $18 \%$ el cual es considerado como un factor importante como complemento en la formación académica. Por consiguiente, Otras herramientas pertenecen a tan solo el $1 \%$ es decir aquel valor no afecta en la aplicación de estrategias.

Los estudiantes están de acuerdo en que se deben analizar ciertas herramientas las cuales pueden ayudar a la mejora del proceso de formación educativa aquellas son los Catálogos y repositorios en línea con un $12.73 \%$ como se observa en la (Figura 3 ), es necesario que en la actualidad estos instrumentos tecnológicos sirvan de ayuda a los estudiantes en la parte de investigación es decir, a enriquecer los conocimientos y que el fácil acceso conjunto con la gratuidad, el ahorro de tiempo al indagar desde la comodidad de sus hogares en estas plataformas eleve el interés del alumnado por el desarrollo de tareas, deberes, consultas, para esto el modelo MIGME permitirá establecer este tipo de estrategias que ayudarán a satisfacer las necesidades del estudiante. Mientras que el $22.87 \%$ corresponde a la misma herramienta anterior la cual es Catálogos y repositorios en línea es decir posee un direccionamiento negativo, con respecto al $64.40 \%$ es un nivel 
de satisfacción favorable en cuanto a percepción de Bibliotecas equipadas adecuadamente.

El alumnado ha señalado que es de gran importancia los puntos del modelo educativo MIGME, se puede observar en la (Figura 4) sin embargo, el grado de satisfacción es insuficiente es decir no está inmerso en los procesos académicos como son las Actividades de enseñanza y educación que corresponde al $8.53 \%$ para ello es necesario implementar estrategias para alcanzar y satisfacer las necesidades estudiantiles, además de promover al grado de relevancia en los puntos mencionados con anterioridad, es de gran importancia en el entorno estudiantil, el cual está enlazado al prestigio de marca y acreditación de la Universidad Técnica de Ambato. Además del Costo - Beneficio es del $22.80 \%$ es un factor trascendental cabe recalcar que posee tendencia a salvaguardar el nivel positivo de satisfacción. Por lo tanto, el $68.67 \%$ que corresponde a dos factores tanto al Bienestar y solución estudiantil como Costo-Beneficio que en actualidad se mantiene positivamente en el progreso universitario.

La importancia los factores críticos del éxito en la Educación Superior (Figura 5), la mayoría de los estudiantes con un resultado del $69.69 \%$ consideran que es relevante, ya que estos factores pueden participar como: incentivos, oportunidades para prepararse y obtener beneficios a futuro, el cual determina un resultado positivo. Por consiguiente, se encuentra en la parte intermedia el $20.44 \%$, esto se debe a que no descartan que estos factores sean necesarios, pero no consideran relevantes en la Educación Superior, el 9,87\% de los estudiantes no determinan importantes dichos factores, este resultado puede reflejarse como una oportunidad para dialogar sobre la utilidad de los mismos.

\section{Implementación de posibles Estrategias de Marketing Educativo - Modelo MIGME para alcanzar el grado de satisfacción del alumnado}

- Estructurar un plan de mejora para determinar los problemas de demora en los procesos estudiantiles, el cual permitirá evaluar constantemente y planificar un mejor esquema, con el fin de mejorar la eficiencia de la atención al alumnado. Establecer un departamento de interacción estudiantil, el cual escuche las necesidades de los estudiantes y proceda a solucionar inquietudes.

- Fortalecer capacitaciones y congresos a través de convenios frecuentes con instituciones públicas y privadas como: Ministerio de Trabajo, Ministerio de Salud, Ministerio de Educación, Contraloría, SRÍ, Plasticaucho, Oscus, Hospital Milenium, con el objetivo de instruir a la comunidad universitaria en diversos ámbitos que refuercen sus carreras y avalen dicha formación mediante certificados, además se requiere la participación del estudiante como refuerzo al diálogo que emita comentarios, dudas, quejas.

- Actualizar catálogos y repositorios en línea a través de una mejor distribución por temas, años de publicación, carreras, autores, para que la búsqueda sea rápida, eficiente, además aquellos sitios web deberían estar enlazados a materiales funcionales como audios, videos que permita reforzar los temas relacionados dado que el estudiante encuentre comprensión y satisfacción al momento de investigar. 
- Renovar actividades de enseñanza y educación a través de la participación dinámica entre docentes y estudiantes es decir que en las clases no exista mucho texto en diapositivas al contario sean ejemplificadas con el análisis de imágenes, casos que tengan relevancia en cada tema tratado a fin de que el alumnado retenga con facilidad y no causar desinterés en el momento del aprendizaje, por lo cual los deberes, consultas se deben debatir en clase con designio a la preparación de cada tema a conocer. Por consiguiente, por medio de un correo electrónico solicitar retroalimentación académica al docente encargado de la asignatura u otro que adquiera el perfil relacionado para conseguir el refuerzo de temas no comprendidos en su totalidad.

- Realizar reuniones estudiantiles, en la cual se permita establecer un diálogo sobre la importancia y como poder obtener beneficios de los factores económicos, académicos y psicosociales de la Educación Superior, con el fin de brindar información para que los estudiantes generen oportunidades a corto o a largo plazo, el cual permitirá generar nuevos conocimientos y aclarar inquietudes.

\section{Conclusiones.}

- En definitiva, el marketing educacional aporta de manera favorable en la Universidad Técnica de Ambato como aquella herramienta que busca posicionarse en la mente del consumidor para alcanzar el nivel máximo de satisfacción y acreditación en la variedad de carreras universitarias. Además, la institución puede determinar aspectos educacionales que pueden ser de mayor utilidad hacia su alumnado.

- De igual manera, se considera que el modelo MIGME permite conocer diversos criterios por parte del alumnado en cuanto a comunicación, intercambio, facilitación, infraestructura, actividades de enseñanza y educación como lo son miembros intervinientes es decir docentes, funcionarios, directivos. Este modelo educativo permite analizar la situación actual de la institución para resaltar los elementos negativos e implementar mejoras para la institución y el estudiante. Este modelo es muy eficiente para diagnosticar las carencias y aspectos que pueden ser mejorados.

- Así entonces, la Universidad Técnica de Ambato busca a través de sus métodos de enseñanza y aprendizaje formar profesionales con conocimientos valederos que aporten al desarrollo organizacional, capaces de solucionar inconvenientes.

- La satisfacción estudiantil es fundamental en la Universidad ya que permite constatar si los procedimientos que se llevan a cabo son favorables o no para el alumnado, además soluciona las inconformidades mediante la búsqueda de una respuesta e implementa de la forma más adecuada. 


\section{Referencias bibliográficas.}

Aldamiz, C. (2016). Marketing de Desarrollo. Madrid : Iepala.

Almanza, K., \& Ustate, M. (2018). Marketing educativo como factor de competitividad en las instituciones de educación superiro. revista.redipe, 206-209.

Andrade, F. (2017). Tutorías de Marketing destruyendo paradigmas en el Ecuador. Guayaquil: liveworking.

Barcia, E., \& Cevallos, N. (2019). Marketing Digital educativo para las instituciones. FIPCAEC , 511-515.

Barreto, C. R. (2016). Las TIC en la Educación Superior, Expeiencias de la Innovación. Barranquilla: Universidad del Norte.

Bassat, L. (2016). El libro rojo de las marcas. China: Random House .

Belío, J. L. (2016). Conozca el Nuevo Marketig. España: Especial Directivos.

Bid, A. (2016). El Plan de Marketing. México: Spanish Edition.

Cárdenas, L. (2015). redalyc. La mercadotécnica en el servicio educativo privado en Mexico, 11.

Dvoskin, R. (2016). Fundamentos de Marketing . Buenos Aires : Granica.

Escobar, P. (2016). Investigación y Educación Superior. Colombia: Universidad Metropolitana de Colombia.

Ferré, A. (2017). Desarrollo y Evaluación de Comptencias en Educación Superior. Madrid: Narcea.

Gonzalez, J. E. (2016). Nuevas tendencias en Innovación de Educación Superior . Madrid España: ACCI.

Grande, I. (2017). Marketing de los Servicios . Madrid : Esic.

Hacker, G. (2017). Marketing. El futuro del Social Media y la Publicidad. México: Social Business.

Jiménez, J. A. (2015). Fundamentos de Marketing . Madrid : Business \& Marketing School-Esic.

Kawasaki, G. (2015). El arte de cautivar. California: Alfa.

Knight, P. (2017). El Profesorado de Educación Superior,Formación para la Excelencia. Narcea .

Kotler, P. (2015). Dirección de Marketing . México: Pearson. 
Larios, E. (2015). Mercadotécnia educativa. CPMARK, 14-17.

Llorente, C. (2019). Marketing educativo, capacitación y fidelización de alumnos. Madrid: ESIC.

Magro, C. (2015). Marketing Educativo . España: Innovación Educativa.

Mamani, E. (2016). Marketing Estrategico relacional Educativo. "Kuskiykuy”, 179-181.

Meneses, J., Muñoz, J., Cantos, S., \& Freire, F. (2018). El marketing en la educación superior en el Ecuador. Contribuciones a las ciencias sociales , 25-34.

Núñez, V. (2017). Marketing educativo: Como comunicar la propuesta de valor de nuestro centro. España: EDICIONES SM.

Ospina, M., \& Sanabria, P. (2010). Un enfoque de me4rcadeo de servicios educativos para la gestión de las organizaciones de e3ducación superior en colombia: Modelo MIGME. fac.cienc, 122-125.

Paris, J. A. (2015). Marketing Estrategico desde el paradigma escencial. Alemania: Alfaomega.

Parmerlee, D. (2017). Las Herramientas de Marketing . Barcelona, España: Granica S.A.

Pastor, V. (2017). Evaluación Formativa y Compartida en Educación Superior. Madrid, España: Narcea.

Pérez, M. S. (2018). Casos de Marketing y Estrategias . Barcelona: UOC.

Rocafuerte, C. (2016). Educación Relevante Supeior . México: Itson .

Rué, J. (2018). El Aprendizaje Autónomo en Educación Superior. Madrid, España: Narcea.

Ruiz, A. (2017). La innovación den Educación Superior. México: Universidad Pedagógica Nacional.

Sanz, D., Crissien, T., García, J., \& Patiño, M. (2016). Marketing educativo como estrategia de negocio de las universidades privadas. unisimon, 161-164.

Sanz, M. L. (2017). Competencias Congnitivas en Educación Superior. Madrid, España: Narcea.

Sarrias, L. (2018). School Marketing. Barcelona: PROFIT.

Soriano, C. (2017). Las Tres Dimensiones del Marketing de Servicios . Madrid España: Diaz de Santos S.A. 
Suysuy, E., \& Taira, D. (2018). Estrategias del Marketing Educativo para el posicionamiento deuna institución educativa 2018. Horizonte Empresarial, 2527.

Tejada, K., \& Reyes, S. (2015). Impacto de implementación de marketing educativo en la gestión de la Universidad del Este. UCE, 9-15.

Tobón, S. (2016). Competencias, Calidad y Educación Supeior. Bogotá, Colombia: Cooperativa Magisterio.

Tzu, S. (2016). El arte de la guerra. Buenos Aires : Lea S.A.

\section{Ciencia}




\section{PARA CITAR EL ARTÍCULO INDEXADO.}

Sánchez Núñez, K. A., Vinueza Barrionuevo, K. M., \& Santamaría Díaz, E. C. (2021). Marketing educacional como factor crítico de éxito en el servicio académico para alumnos universitarios . Visionario Digital, 5(3), 6-28. https://doi.org/10.33262/visionariodigital.v5i3.1744

\section{LCiencia}

El artículo que se publica es de exclusiva responsabilidad de los autores y no necesariamente reflejan el pensamiento de la Revista Visionario Digital.

El artículo queda en propiedad de la revista y, por tanto, su publicación parcial y/o total en otro medio tiene que ser autorizado por el director de la Revista Visionario Digital.
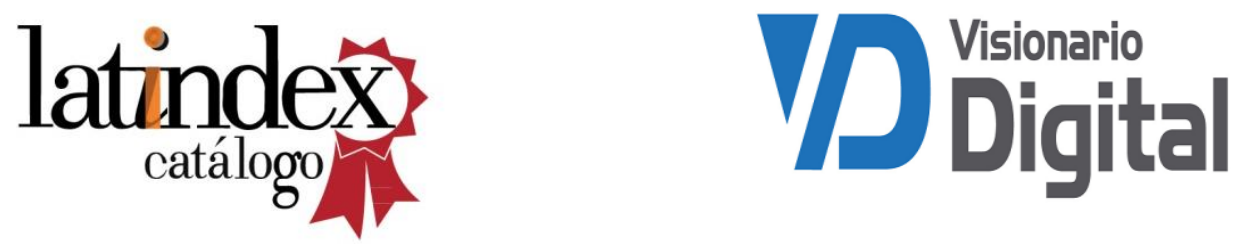